\title{
Overcome the Energy Gap Law in Near-Infrared Emissive Aggregates via Nonadiabatic Coupling Suppression
}

\author{
Jie Xue, ${ }^{1,2}$ Jingyi Xu,' Jiajun Ren,, ,* Qingxin Liang, ${ }^{1}$ Qi Ou, ${ }^{1}$ Rui Wang, ${ }^{1}$ Zhigang Shuai, ${ }^{2}$ and \\ Juan Qiao ${ }^{1,2,3, * *}$ \\ 'Key Lab of Organic Optoelectronics and Molecular Engineering of Ministry of Education, Department of Chemistry, Tsinghua University, Beijing, \\ 100084, P. R. China \\ ${ }^{2}$ Center for Flexible Electronics Technology, Tsinghua University, Beijing 100084, P. R. China \\ 3Lead Contact \\ *Correspondence: renjj@tsinghua.edu.cn \\ ${ }^{* *}$ Correspondence: qjuan@mail.tsinghua.edu.cn
}

\begin{abstract}
The pursuing of purely organic materials with high-efficiency nearinfrared (NIR) emissions is fundamentally limited by the large nonradiative decay rates $\left(k_{n r}\right)$ governed by the energy gap law. Here, we demonstrated a feasible and innovative strategy by employing intermolecular charge-transfer (CT) aggregates (CTA) to realize highefficiency NIR emissions via nonadiabatic coupling suppression. The formation of CTA engenders intermolecular CT in the excited states; thereby, not only reducing the electronic nonadiabatic coupling and contributing to small $k_{n r}$ for high-efficiency NIR photoluminescence, but also stabilizing excited-state energies and achieving thermally activated delayed fluorescence for high-efficiency NIR electroluminescence. This work provides new insights into aggregates and opens a new avenue for organic materials to overcome the energy gap law and achieve high-efficiency NIR emissions.
\end{abstract}

Nonadiabatic coupling, intermolecular charge-transfer, near-infrared, molecular aggregates, organic light-emitting diodes, thermally activated delayed fluorescence

\section{INTRODUCTION}

Harnessing the near-infrared (NIR) light is of great importance for optogenetics, phototherapy, bio-imaging, medical diagnosis and optical communications..$^{1-5}$ In recent years, NIR organic materials have attracted rapidly growing interest because of their potential as efficient, low-toxicity, low-cost and even disposable NIR photosensitizer and

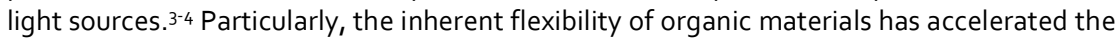
development of NIR organic light-emitting diodes (OLEDs) as flexible, wearable and implantable devices.4, 6-9 Extending $\pi$-conjugation length and employing donor-acceptor charge transfer state have proven to be effective molecular design strategies to realize NIR emissions. ${ }^{3}$ However, these conventional NIR organic molecules usually suffer from large non-radiative decay rates $\left(k_{\mathrm{nr}}\right)$ and low photoluminescence $(\mathrm{PL})$ quantum yields (PLQY) due to the energy gap law, $4,10-11$ which dictates accelerated non-radiative processes from the lowest excited state $\left(S_{1}\right)$ to ground state $\left(S_{0}\right)$ caused by molecular vibrations as the optical energy gap falls towards deep-red and NIR region. Therefore, high-efficiency NIR-OLEDs are still rare, even though great breakthroughs have been made for purely organic materials to harvest the weakly emissive triplet excitons generated by electroexcitation. ${ }^{12-15}$
The bigger picture

Overcome the energy gap law! Invisible near-infrared (NIR) light plays a highly visible role in biomedical sciences, photovoltaics and optical communications, benefiting human life. Though urgently needed for flexible, wearable and implantable applications, NIR organic materials suffer from accelerated non-radiative decay rates $\left(k_{\mathrm{nr}}\right)$ and the resulted poor luminous efficiency as governed by the energy gap law. To date, reported endeavors to decelerate $k_{n r}$ are mainly focusing on reducing the electron-vibration coupling with the electronic nonadiabatic coupling (NAC) assumed as a constant. Here, we uncover that the suppression of NAC is a novel approach to decelerate $k_{\mathrm{nr}}$ and overcome the energy gap law. Also, a feasible strategy was demonstrated by forming intermolecular charge-transfer aggregates (CTA) to suppress NAC and thus achieve highefficiency NIR emission. This work provides a new avenue for organic materials to overcome the energy gap law. 
To alleviate the limitation imposed by the energy gap law, considerable efforts have been made for organic materials to decelerate the non-radiative decay processes by reducing the electron-vibration coupling. At single-molecule level, employing rigid molecular structure can hinder low-frequency vibrations in molecules, and perdeuterated or perfluorinated approaches can alleviate high-frequency vibrations. ${ }^{16-18}$ Nevertheless, those strategies would inevitably increase the difficulty and cost of synthesis. Beyond the single-molecule level, advances in understanding the aggregation effect on organic luminescence indicate that intermolecular packing sometimes can hinder the non-radiative decay processes caused by low-frequency vibrations of functional groups. ${ }^{19-20}$ However, there is still a large room for the improvement of the luminescence efficiency of NIR organic materials, which requires deeper insights into the energy gap law.

In this work, from a fundamental perspective on the energy gap law, we found the suppression of electronic nonadiabatic coupling is a novel approach for organic emissive materials to decelerate the non-radiative decay processes and overcome the energy gap law. Furthermore, we demonstrated a feasible strategy by employing intermolecular chargetransfer (CT) aggregates (CTA) to suppress the nonadiabatic coupling and achieve highefficiency NIR emissions. The formation of CTA with the involvement of intermolecular CT $(\mathrm{XCT})$ in the excited states can not only reduce the nonadiabatic coupling, which leads to a small $k_{\mathrm{nr}}$ for high-efficiency NIR photoluminescence, but also stabilize excited-state energies, which induces strong thermally activated delayed fluorescence (TADF) for high-efficiency NIR electroluminescence (EL). The developed purely organic CTA emitter exhibited widelytunable emissions from 621 to $802 \mathrm{~nm}$ with high PLOYs of over $80 \%$ in NIR region and high maximum external quantum efficiencies (EQEs) of $17.0 \%$ in NIR-OLEDs.

\section{RESULTS}

\section{Theoretical Analysis and Conceptual Design}

First, we briefly review the theoretical background of the non-radiative decay rate $k_{\mathrm{nr}}$. For organic molecules in the weak coupling limit (Huang-Rhys factor $S_{i} \ll 1$ ), $k_{\mathrm{nr}}$ can be expressed as ${ }^{11}$

$$
k_{\mathrm{nr}}=\sum_{l} C_{l}^{2} \frac{\sqrt{2 \pi}}{\sqrt{\omega_{\mathrm{M}} \Delta E}} \exp \left\{-\sum_{\mathrm{i}} S_{i}\right\} \exp \left\{-\frac{\Delta E}{\omega_{\mathrm{M}}}\left[\ln \left(\frac{\Delta E}{\lambda_{\mathrm{M}}}\right)-1\right]\right\}
$$

The prefactor $C_{l}^{2}$ is related to the nonadiabatic coupling elements (NACE) between the ground state $\varphi_{\mathrm{gs}}$ and the excited state $\varphi_{\mathrm{ex}}$ along mode $l$, which is called the promoting mode triggering the electronic transition.

$$
C_{l}^{2}=\left|\left\langle\varphi_{\mathrm{ex}}\left|\frac{\partial}{\partial q_{l}}\right| \varphi_{\mathrm{gs}}\right\rangle\right|^{2} \frac{\omega_{l}}{2}
$$

The other parts of Eq. (1) are related to the Franck-Condon factor weighted density of states - the overlap between the initial and final vibrational wavefunctions, which represents the ability of vibrations to receive the electronic energy. $\omega_{M}$ is the frequency of the normal vibrations of maximum frequency. $\lambda_{\mathrm{M}}$ is the total reorganization energy of the vibrations with frequency $\omega_{\mathrm{M}} . \Delta E$ is the adiabatic energy gap between the electronic ground and excited states. Commonly, it is believed that $k_{\text {nr }}$ would increase exponentially with the decrease of $\Delta E$ under the assumption that the prefactor $C_{l}^{2}$ is unchanged. This is the widely known energy gap law and mainly hinders the development of high-efficiency NIR materials.

From Eq. (1), it is clear that the $k_{\mathrm{nr}}$ of organic molecules are determined by two factors, the electronic nonadiabatic coupling and the Franck-Condon factor weighted density of states. To date, strategies for organic materials to reduce $k_{\mathrm{nr}}$ are mainly focusing on suppressing the second part of Eq. (1) by reducing $\lambda_{\mathrm{M}}$ and $\omega_{\mathrm{M}} \cdot{ }^{16-22}$ Notably, Zhou and Chi et al. proposed that exciton delocalization in well-aligned $\mathrm{Pt}(\mathrm{II})$ complexes can reduce the reorganization energy $\lambda_{M}$ and achieve high-efficiency NIR-OLEDs. ${ }^{22}$ Besides the suppression of electronvibration coupling, the reduction of electronic nonadiabatic coupling is another approach to reduce $k_{\mathrm{nr}}$ and overcome the energy gap law. However, the prefactor $C_{l}^{2}$ corresponding to the electronic NACE is commonly assumed as a constant and its effect is rarely discussed. Within the complete basis set limit, ${ }^{23}$ the NACE is qualitatively proportional to the electronic transition density, determined by the overlap between the electron and hole wavefunction as shown in Eqs. (3) and (4).

$$
\begin{aligned}
\left\langle\varphi_{\mathrm{ex}}\left|\frac{\partial}{\partial q_{l}}\right| \varphi_{\mathrm{gs}}\right\rangle \approx \frac{1}{E_{\mathrm{ex}}-E_{\mathrm{gs}}} \sum_{\sigma} \frac{z_{\sigma} e^{2}}{\sqrt{M_{\sigma}}} \sum_{i=x, y, z} E_{\sigma i} L_{\sigma i, l} & \text { (Equation 3) } \\
E_{\sigma i}=\int d \vec{r} \rho(\vec{r}) \frac{e\left(r_{i}-R_{\sigma i}\right)}{\left|\vec{r}-\vec{R}_{\sigma}\right|^{3}} \approx \int d \vec{r} \phi_{\mathrm{elec}}(\vec{r}) \phi_{\mathrm{hole}}(\vec{r}) \frac{e\left(r_{i}-R_{\sigma i}\right)}{\left|\vec{r}-\vec{R}_{\sigma}\right|^{3}} & \text { (Equation 4) }
\end{aligned}
$$


where $L_{\sigma i, l}$ is the transformation matrix between the normal coordinates and Cartesian coordinates and $E_{\sigma i}$ is atomic electric field experienced by the overlap of electron and hole from the excited state. From Eqs. (1)-(4), we propose that the decrease of the overlap of electron and hole in the excited states can reduce the prefactor $C_{l}^{2}$, thus suppressing the nonradiative decay processes for a small $k_{n r}$. Interestingly, this coincides with the design strategy of TADF materials, which needs to minimize the energy gaps ( $\left.\Delta E_{S T}\right)$ between the $\mathrm{S}_{1}$ and lowest triplet $\left(T_{1}\right)$ excited states by decreasing the overlap of electron and hole of the excited states.

\section{Molecular Design and Synthesis}

Recently, we found the formation of J-aggregates with strong $\mathrm{XCT}$ can significantly stabilize the excited-state energies of organic materials, thus inducing and enhancing highly efficient TADF. ${ }^{24}$ Most importantly, the formation of such molecular aggregates can lead to a decreased $k_{\mathrm{nr}}$ with the decrease of the optical energy gap, totally deviating from the exponential increase trend imposed by the energy gap law. ${ }^{24}$ Although the underlying mechanism was unclear at that time, it could benefit from the nonadiabatic coupling suppression induced by $\mathrm{XCT}$ with the larger separation of electron and hole than that of intramolecular CT (iCT). To illustrate the underlying mechanism and achieve high-efficiency NIR emissions, the molecule dpTPAAP (Figure 1) for CTA was designed based on the reported TPAAP with the same electron acceptor acenaphtho[1,2-b]pyrazine-8,9dicarbonitrile (AP) and modified electron donor bis(biphenyl-4-yl)phenylamine (dpTPA). ${ }^{24}$ The addition of phenyl groups could increase the delocalization of the highest occupied molecular orbital (HOMO), which would be conducive to red-shifted emission with high PLOY. ${ }^{25-26}$ And the similar donor-acceptor framework could endow dpTPAAP with strong xCT in aggregation states to construct CTA for NIR emission. For comparison, an iCT molecule with similar molecular framework, dpTPAAZ was designed by fusing electron acceptor pyrazine on the acceptor moiety of dpTPAAP to enhance the ICT and realize NIR emission in single molecule. The fusion of rigid pyrazine could maintain the molecular rigidity, thus reducing the extra non-radiative decay processes as much as possible. The comparative investigations on photophysical behaviors of CTA and commonly-used iCT molecules were conducted to uncover the distinct underlying mechanism and explore the rational design of high-efficiency NIR organic materials.

Both dpTPAAP and dpTPAAZ were synthesized in three steps involving bromination, SuzukiMiyaura cross-coupling reaction and dehydration cyclization reaction. These new compounds were characterized by NMR spectroscopy, high-resolution mass spectrometry, and elemental analysis. Thermogravimetric analysis (TGA) measurements revealed that both dpTPAAP and dpTPAAZ have high decomposition temperatures ( $T_{\mathrm{d}}$, corresponding to $5 \%$ weight loss) at 414 and $498^{\circ} \mathrm{C}$, respectively.

\section{Single Crystal and Molecular Packing}

During temperature gradient vacuum sublimation, single crystals of dpTPAAP were readily obtained. The molecular structure and packing motifs of dpTPAAP in crystalline states were identified by single-crystal X-ray crystallographic analysis. The dpTPAAP molecules possess relatively planar structures and the dihedral angle between donor and acceptor subunits is $33^{\circ}$ (Figure $2 \mathrm{~A}$ ). In crystalline state, the nearest neighbouring dpTPAAP molecules form dimers in a face-to-face fashion (Figure $2 \mathrm{~B}$ ) with the donor of one molecule close to the acceptor of the other molecule, which are supported by intermolecular $\mathrm{CN} \cdots \mathrm{H}-\mathrm{C}$ interactions with a distance of $2.886 \AA$ and intermolecular $\pi \cdots \pi$ interactions with a distance of $3.521 \AA$ between the AP acceptor cores. These head-to-tail dimers pack up in a herringbone arrangement in the crystal (Figure $2 \mathrm{C}$ ), which is much different from the staircase packing mode of its counterpart TPAAP. ${ }^{24}$

\section{Photophysical Properties}

To study the photophysical properties of these compounds as single molecules, oxygen-free dilute toluene solutions $\left(1 \times 10^{-5} \mathrm{~mol} \mathrm{~L}^{-1}\right)$ of dpTPAAP and dpTPAAZ were prepared. As shown in Figure $3 \mathrm{~A}$, the intense absorption bands below $430 \mathrm{~nm}$ are assigned to the localized $\pi-\pi^{*}$ transitions and the moderate absorption bands over $430 \mathrm{~nm}$ are ascribed to the iCT transitions. The broad iCT absorption band of dpTPAAZ can be deconvoluted (Gaussian multi-peak fitting) into two absorption bands (Figure S2); the high-energy band with peak at $466 \mathrm{~nm}$ corresponds to the second lowest excited single state $\left(S_{2}\right)$, and the low-energy band with peak at $538 \mathrm{~nm}$ corresponds to $S_{1}$, which is red-shifted compared with that of dPTPAAP 
with the peak at $501 \mathrm{~nm}$ due to the enhancement of iCT. Under photoexcitation, dpTPAAP displays intense red fluorescence $\left(\lambda_{\max }=624 \mathrm{~nm}\right)$ with a high PLQY of up to $97.4 \%$ and a short lifetime of $7.94 \mathrm{~ns}$ (Figure $S_{3}$ ). The remarkable PLOY results from high $k_{\mathrm{r}}$ value of $1.2 \times 10^{8} \mathrm{~s}^{-1}$ and low $k_{n r}$ value of $3.3 \times 10^{6} \mathrm{~s}^{-1}$. As expected, thanks to the enhancement of iCT, dpTPAAZ exhibits largely red-shifted emission into desired NIR region $\left(\lambda_{\max }=763 \mathrm{~nm}\right)$ in toluene solution. Unfortunately, such large red-shift of emission leads to a dramatically reduced PLQY to $7.6 \%$. The low PLOY of dPTPAAZ results from the low $k_{r}$ value of $1.8 \times 10^{7} \mathrm{~s}^{-1}$ and high $k_{n r}$ value of $2.2 \times 10^{8} \mathrm{~s}^{-1}$. In comparison with dpTPAAP, dpTPAAZ showed almost one order of magnitude smaller $k_{\mathrm{r}}$ value and two order of magnitude larger $k_{\mathrm{nr}}$ value, clearly manifesting that the photophysical behaviours of single organic molecule is severely limited by the energy gap law.

In addition to enhancing $\mathrm{iCT}$ at the level of single molecule, the formation of aggregates with strong XCT could also achieve desired red-shift of emission. ${ }^{24,} 27-28$ In neat film, dpTPAAP shows desired NIR emission ( $\lambda_{\max }=802 \mathrm{~nm}$ ), with a $178 \mathrm{~nm}$ red-shift compared with that in toluene solution. The neat film gives a remarkable PLOY of $13.0 \%$, which is more than three times that of the curcuminoid derivative reported by Adachi et al $\left(\lambda_{\max }=801 \mathrm{~nm}\right.$ and PLQY $=$ $4 \%) .{ }^{29}$ Also, the CT absorption band of the neat film shows a substantial red-shift $(\sim 40 \mathrm{~nm})$ with corresponding peak wavelength locating at $541 \mathrm{~nm}$, which indicates the formation of molecular aggregates with strong $\times C T .{ }^{24}$ The single crystals of dpTPAAP demonstrated further red-shifted absorption and emission $\left(\lambda_{\max }=882 \mathrm{~nm}\right)$ compared with its neat film (Figure $3 A$ ), suggesting a more significant $x C T$ in single crystals. Importantly, dpTPAAP single crystal shows a good PLQY of $4.4 \%$.

To investigate the influences of molecular aggregation and environmental dipole on exciton states of dpTPAAP, we measured the absorption spectra of dpTPAAP in tetrahydrofuran $(\mathrm{THF})$ /water mixtures with different water volume fractions $\left(f_{w}\right)$. As shown in Figure $3 \mathrm{~B}$, although the polarity of THF/water mixture rises as the increase of $f_{w}$ from $0 \%$ to $50 \%$, the absorption spectra of dpTPAAP is almost unchanged, thus excluding the possibility of environmental dipole induced large red-shift of absorption. The further increase of $f_{\mathrm{w}}$ from $50 \%$ leads to an abrupt red-shift of CT absorption band accompanied by the formation of nanoparticles with the peak finally pinned at $532 \mathrm{~nm}$, manifesting that the formation of molecular aggregates is the main cause for the large red-shift of CT absorption band, which greatly stabilizes the exciton states.

To explore the evolution of molecular aggregates in solid states, we studied the photophysical properties of doped films with dpTPAAP in 2,2',2"-(1,3,5-benzinetriyl)-tris(1phenyl-1-H-benzimidazole) (TPBi) at different doping concentrations. As shown in Figure ${ }_{3} \mathrm{C}$, the absorption spectra of doped films show progressive red-shift with increasing doping concentration, manifesting the gradual formation of molecular aggregates. Meanwhile, these films demonstrate widely-tunable PL by simply controlling the doping concentration (Figure $3 \mathrm{D}$ and $3 \mathrm{E}$ ). At very low doping concentration of $0.1 \mathrm{wt} \%$, the doped film displays strong red PL $\left(\lambda_{\max }=621 \mathrm{~nm}\right)$, which is similar to the PL of dpTPAAP in dilute toluene solutions. As the increase of doping concentration, $0.5 \mathrm{wt} \%$ and $1 \mathrm{wt} \%$ doped film give strong deep-red (DR) emissions at $653 \mathrm{~nm}$ and $677 \mathrm{~nm}$ with very high PLQYs of $92.1 \%$ and $89.4 \%$, respectively. When doping concentration increases to $5 \mathrm{wt} \%$, the doped film displays desired NIR emission $\left(\lambda_{\max }=707 \mathrm{~nm}\right.$ ) with a high PLOY of $82.2 \%$. The doped films show persistently red-shifted $\mathrm{PL}$ as the doping concentration continuously increases.

Notably, the $30 \mathrm{wt} \%$ doped film realizes the desired NIR PL with peak wavelength at $760 \mathrm{~nm}$, which is almost the same to the PL $\left(\lambda_{\max }=763 \mathrm{~nm}\right)$ of dpTPAAZ molecules in toluene. Moreover, the $30 \mathrm{wt} \%$ doped film has an impressive PLOY of up to $38.5 \%$, which is 5 times that of dPTPAAZ molecules in toluene. Such remarkable PLOY benefits from its high $k_{\mathrm{r}}$ value of $2.26 \times 10^{7} \mathrm{~s}^{-1}$ and low $k_{\mathrm{nr}}$ value of $3.60 \times 10^{7} \mathrm{~s}^{-1}$. The $k_{\mathrm{r}}$ value is 1.2 times that of dpTPAAZ molecules in tolvene, which indicates that the formation of such molecular aggregates is favourable to obtain high $k_{\mathrm{r}}$ values. Importantly, the film displays a substantially reduced $k_{\mathrm{nr}}$ value by about one order of magnitude compared with that of IPTPAAZ molecules in oxygen-free tolvene. This demonstrates that the formation of CTA could significantly suppress the non-radiative decay processes and contribute to a small $k_{n r}$ value rather than a rapid increased $k_{n r}$ value according to the energy gap law. Meanwhile, the $30 \mathrm{wt} \% \mathrm{film}$ exhibits prominent TADF consisting of a nanosecond-scale prompt component (average lifetime $\tau_{p}=16.3 \mathrm{~ns}$ ) and a microsecond-scale delayed component (average lifetime $\tau_{\text {TADF }}=$ 
$39.4 \mu \mathrm{s}$ ) at room temperature (Figure $3 \mathrm{~F}$ and Figure S6), which could be ascribed to the substantially reduced $\Delta E_{\mathrm{ST}}$ of only $0.073 \mathrm{eV}$ as the result of molecular aggregation and the self-polarization-induced solid-state solvation effect. ${ }^{24,30}$

The photophysical properties of ApTPAAZ in the doped films were also studied for comparison. The $5 \mathrm{wt} \%$ dpTPAAZ:TPBi film displays NIR emission with peak wavelength at $764 \mathrm{~nm}$ (Figure S7) and a low PLQY of 5.9\%, which are close to those of dpTPAAZ molecules in toluene $\left(\lambda_{\max }=763 \mathrm{~nm}, \mathrm{PLOY}=7.6 \%\right)$. From dilute toluene solution to $5 \mathrm{wt} \%$ doped film, the $k_{\mathrm{nr}}$ value of dpTPAAZ reduces from $2.2 \times 10^{8} \mathrm{~s}^{-1}$ to $1.4 \times 10^{8} \mathrm{~s}^{-1}$, which could be ascribed to the suppression of low-frequency molecular motions in solid state. Remarkably, the $30 \mathrm{wt} \%$ dPTPAAP:TPBi film has almost the same emission $\left(\lambda_{\max }=760 \mathrm{~nm}\right)$ to that of the $5 \mathrm{wt} \%$ dpTPAAZ:TPBi film, but its $k_{\mathrm{nr}}$ value is only 0.26 times that of the latter. In general, the doped films with high doping concentration would exhibit large $k_{n r}$ caused by aggregation-caused quenching. The unexpected low $k_{n r}$ value of $30 \mathrm{wt} \%$ dpTPAAP:TPBi film could benefit from the formation of CTA. Consequently, the PLQY of $30 \mathrm{wt} \%$ dpTPAAP:TPBi film is 6.5 times that of $5 w t \%$ dpTPAAZ:TPBi film.

\section{Electron Paramagnetic Resonance}

To investigate the $\mathrm{xCT}$ in the aggregation states, we performed electron paramagnetic resonance (EPR) measurements on the single crystals and neat film of dPTPAAP. As shown in Figure $4 \mathrm{~A}$, the single crystals of dpTPAAP under dark condition displayed clear EPR signal with the $g$-factor determined to be 2.0033 . Under photoexcitation, its EPR signal is slightly enhanced. Unlike the single crystal with long-range order, the neat film of dpTPAAP is more likely to be amorphous (Figure Sg). Nevertheless, the neat film of dpTPAAP also displayed EPR signal under dark condition with the $g$-factor determined to be 2.0040 (Figure $4 \mathrm{~B}$ ). Under photoexcitation, its EPR signal is remarkably enhanced instead. Both the $g$-factors of EPR signals of single crystals and neat film are close to the value of free electron (2.0023), indicating the presence of unpaired electrons. Since dpTPAAP is a closed-shell neutral molecule, the unpaired electrons could be ascribed to the charged ions generated by XCT in the ground state due to the formation of CTA. ${ }^{27,}{ }^{31}$ The significantly enhanced EPR signal of neat film under photoexcitation demonstrates that more charged ions are generated by $x C T$ under photoexcitation, which clearly highlights the $\mathrm{xCT}$ nature in the excited states. In comparison, the photoexcitation only led to slightly enhanced EPR signal for dpTPAAP single crystals, which could be ascribed to the opacity of single crystals that allows only a small ratio of molecules photoexcited under illumination.

\section{Quantum Chemical Calculations}

To understand the photophysical behaviours of dPTPAAP, we carried out theoretical investigations on the electronic structures of monomer and dimers in the gas phase using density functional theory (DFT) and time-dependent DFT (TDDFT). An optimal tuning version of $\omega B$ 97XD long-range corrected functional $(\omega=0.1264)$ was used to describe the CT excited states..$^{2}$ The $S_{1}$ and $T_{1}$ energies of the monomer at the optimized $S_{0}$ geometry were calculated to be $2.89 \mathrm{eV}$ and $2.20 \mathrm{eV}$, respectively. According to the analysis of attachment (electron)/detachment (hole) density and fragment-based transition density matrices (TDM) of $S_{1}$ and $T_{1}$ state calculated by Q-Chem and Multiwfn 3.7 package, $33-35$ the $S_{1}$ state of the monomer is predominantly an $\mathrm{iCT}$ state from the donor fragment to the acceptor fragment, while the $T_{1}$ state is mainly a local excitation state in the acceptor fragment (Figure $5 \mathrm{~A}$ and $\left.{ }_{5} \mathrm{~B}\right)$.

To investigate the aggregation effect on the electronic structure, we extracted 14 nearestneighbour dimers from the single crystal (Figure $\mathrm{S}_{10}$ ). Among them, dimer 1 with a face-toface stacking leads to the most significant reductions of $S_{1}$ and $T_{1}$ energies (Table $S_{4}$ ), and the $S_{1}$ and $T_{1}$ energies are $2.31 \mathrm{eV}$ and $1.95 \mathrm{eV}$ respectively at the optimized So geometry. While the $S_{1}$ and $T_{1}$ energies of other dimer are close to those of monomers. Figure ${ }_{5} C$ shows that unlike the case of monomer, the $S_{1}$ state of dimer 1 is mainly composed of XCT and mixed with a small iCT component. To the contrary, $\mathrm{T}_{1}$ state maintains the local excitation feature of monomer and is mixed with a small $x C T$ component. This different contributions of XCT component into $S_{1}$ and $T_{1}$ states result in a different amount of reduction of $S_{1}$ and $T_{1}$ energies upon aggregation $\left(\Delta E_{\mathrm{S}_{1}}=0.58 \mathrm{eV}, \Delta E_{\mathrm{T}_{1}}=0.25 \mathrm{eV}\right)$. Therefore, the singlet-triplet gap decreases in favour of TADF in the solid phase. 
Apart from the contribution of $x \mathrm{CT}$, the excitonic coupling is another possible effect to account for the aggregation induced spectrum shift. After Boys-localized diabatization carried out in the developmental version of $\mathrm{Q}$-Chem within the lowest 4 singlet excited states (Figure $5 \mathrm{D}$ ), it is clear to see that they are composed of 2 degenerate low-energy pure $\mathrm{XCT}$ states and 2 degenerate high-energy pure $\mathrm{ICT}$ states. The large electronic coupling $\left\langle\mathrm{xCT}_{1}|H| \mathrm{iCT}_{2}\right\rangle=\left\langle\mathrm{xCT}_{2}|H| \mathrm{iCT}_{1}\right\rangle=-103 \mathrm{meV}$ may help the weakly emitting $\mathrm{xCT}$ state borrow the oscillator strength from the iCT state to enhance $k_{\mathrm{r}}$ if the ideal degeneracy is broken with static disorder in the real film. However, the excitonic coupling between the iCT states is only $26 \mathrm{meV}$, which has a minor effect on the $S_{1}$ state of dimer 1.

The introduction of $\mathrm{xCT}$ component into the excited states not only reduces the excitation energy, but also reduces NACE, which is essential to the high-efficiency NIR luminescence. In CTA, we found that the reduction of NACE plays a major role in suppressing non-radiative decay process when the $S_{1}$ state changes from $\mathrm{iCT}$ to $\times C T$ upon aggregation. In Figure $6, C_{l}$ in Eq. (1) for different promoting modes of dpTPAAP monomer and dimer 1 are shown. For the monomer, the modes between $1300 \mathrm{~cm}^{-1}$ and $1600 \mathrm{~cm}^{-1}$, typically corresponding to the high-frequency stretching vibrations of skeleton bonds, contribute most to the prefactor. In contrast, for dimer $\mathbf{1}, C_{l}$ in the same frequency window is largely suppressed. The largest component decreases from $40 \mathrm{~cm}^{-1}$ to $15 \mathrm{~cm}^{-1}$. This reduction is due to that the NACE is qualitatively proportional to the overlap between the hole and electron wavefunction as shown in Eq. (4). Though we only consider the minimal dimer model in our calculation, we expect that in the thin film, aggregates larger than the dimer will further delocalize the wavefunction of hole and electron and lead to a smaller NACE. The reduction of the prefactor $C_{l}^{2}$ will partially compensate for the increase in $k_{\mathrm{nr}}$ induced by the reduction in energy gap. It could account for the much smaller $k_{\mathrm{nr}}$ of CTA compared with that of iCT molecules with similar emission wavelength and molecular structure, and could be the underlying mechanism for the observation in the experiments that the $k_{n r}$ shows even an anti energy gap law behaviour through the formation of molecular aggregates with strong $x \mathrm{CT} .{ }^{24}$ The similar effect has been discussed in organic solar cells that the electron-hole separation and delocalization in the donor-acceptor interface will reduce the nonadiabatic coupling and hence suppress the non-radiative decay process. ${ }^{36}$

\section{Device Performance}

To evaluate the electroluminescent performance of dpTPAAP, OLEDs were fabricated with multilayer configurations of indium tin oxide (ITO)/1,4,5,8,9,11hexaazatriphenylenehexacarbonitrile (HATCN, $5 \mathrm{~nm}$, hole-injecting layer)/N,N ' bis(naphthalen-1-yl)-N, $N^{\prime}$-bis(phenyl)benzidine (NPB, $70 \mathrm{~nm}$, hole transporting layer) $/ 4,4^{\prime}, 4^{\prime \prime}$ -tris(carbazol-9-yl)triphenylamine (TCTA, $10 \mathrm{~nm}$, exciton blocking layer)/TPBi: $x$ wt\% dpTPAAP (30 nm, emitting layer)/TPBi $(60 \mathrm{~nm}$, electron transporting layer)/LiF (1 nm, electron-injecting layer)/Al (150 nm) (device A: $x=1$, device $\mathrm{B}: x=5$, device $\mathrm{C}: x=15$ and device $\mathrm{D}: x=30$ ). As depicted in Figure 7 and Table 1 , device A demonstrated intense DR emission with peak wavelength at $675 \mathrm{~nm}$. As the increase of doping concentrations, the EL spectra of devices red-shift and fall into NIR region with peak wavelengths at $710 \mathrm{~nm}, 730 \mathrm{~nm}$ and 752 $n m$ for devices $B, C$ and $D$, respectively. In addition to the widely-tunable EL by simple doping concentration control, devices $A, B, C$ and $D$ based on dpTPAAP achieved high maximum EQEs of $24.8 \%, 17.0 \%, 13.0 \%$ and $6.8 \%$, respectively, which may represent the best results for DR and NIR OLEDs based on TADF emitters. ${ }^{24,29,37-41}$ Importantly, NIR devices B, C and D exhibited high radiant emittances of $69.06 \mathrm{~W} \mathrm{~m}^{-2}, 86.02 \mathrm{~W} \mathrm{~m}^{-2}$, and $78.36 \mathrm{~W} \mathrm{~m}^{-2}$, respectively. As the increase of doping concentration, the turn-on voltage decrease from $3.0 \mathrm{~V}$ for device A to $2.6 \mathrm{~V}$ for device $\mathrm{B}$ and to $2.5 \mathrm{~V}$ for devices $\mathrm{C}$ and $\mathrm{D}$, which could be ascribed to the trapassisted recombination on the dopants at high doping concentration. In addition, devices with higher doping concentration demonstrate a more rapid increase of current density $(J)$ as the increase of voltage, which could be ascribed to the better carrier injection and the formation of percolative charge transport on dopants. ${ }^{42}$ As the increase of doping concentration in the emitting layer, devices show alleviated efficiency roll-offs, which could be ascribed to the shortened delayed fluorescence lifetimes for doped films with higher doping concentration, ${ }^{24}$ thus reducing the exciton-exciton annihilation and exciton-polaron annihilation at high current density.43-44 For comparison, Device E based on dpTPAAZ was also fabricated with a configuration of ITO/ HATCN $(5 \mathrm{~nm}) / \mathrm{NPB}(70 \mathrm{~nm}) / \mathrm{TCTA}(10 \mathrm{~nm}) / 5 \mathrm{wt} \%$ dpTPAAZ:TPBi $(30 \mathrm{~nm}) / \mathrm{TPBi}(60 \mathrm{~nm}) / \mathrm{LiF}(1 \mathrm{~nm}) / \mathrm{Al}(150 \mathrm{~nm})$. Device E demonstrates NIR emission with peak wavelength at $757 \mathrm{~nm}$, which is close to EL peak of device $D$ based on 30 
$w t \%$ dpTPAAP:TPBi film. Nevertheless, the maximum EQE of device $E$ is only $1.2 \%$ due to the low PLQY of the emitting layer, which is much lower than that of device D. Although with nearly the same emission peak, the much higher EQE of device $D$ than device $E$ suggests the superiority of CTA in achieving high-efficiency NIR EL.

\section{DISCUSSION}

In this contribution, we have presented that the suppression of electronic nonadiabatic coupling is a novel approach for organic emissive materials to suppress the non-radiative decay processes and overcome the energy gap law. Also, a feasible strategy was developed to reduce the electronic nonadiabatic coupling by forming CTA for organic materials to achieve high-efficiency NIR emissions. The developed dpTPAAP molecules, through forming CTA, exhibit widely-tunable emissions from 621 to $802 \mathrm{~nm}$ with high PLOYs of over $80 \%$ in NIR region. Importantly, in comparison with iCT molecules with similar molecular structure and NIR emission around $760 \mathrm{~nm}$, the CTA emitter displays a 6.5 times higher PLOY contributed by a 3.8 times smaller $k_{n r}$. Theoretical investigations reveal that the formation of CTA with the involvement of $X C T$ in the excited states can suppress nonadiabatic coupling, thus alleviating the limitation of energy gap law. The formation of CTA is well verified by the absorption spectra and EPR measurements and rationalized by quantum chemical calculations. The OLEDs fabricated with the CTA emitter exhibited high-efficiency DR and NIR EL. The mechanism illustrated by the theoretical investigations would shed light on the unique photophysical behaviours of $\mathrm{xCT}$ emitters and provide a novel approach for organic materials to overcome the energy gap law. It is anticipated that our work paves a superior way for the rational design of high-efficiency NIR organic materials, and lays a foundation for the applications of CTA for a wide variety of fields.

\section{EXPERIMENTAL PROCEDURES}

\section{Resource availability}

\section{Lead contact}

Further information and requests for resources should be directed to and will be fulfilled by the lead contact, Juan Qiao (qjuan@mail.tsinghua.edu.cn)."

Materials availability

All materials generated in this study are available from the lead contact without restriction

Data and code availability

The accession number for the crystal structure CIF reported in this paper is CCDC: 1975023 (dpTPAAP).

\section{Materials and Synthesis}

All reagents and chemicals were reagent grade, commercially available and used without further purification. OLED materials HATCN, NPB, TCTA and TPBi were purchased from Xi'an Polymer Light Technology Corp and were used as received. ${ }^{1} \mathrm{H}$ and ${ }^{13} \mathrm{C}$ NMR spectra were measured on a JEOL ECA 600 spectrometer at ambient temperature. The laser desorption ionization time-of flight mass spectrometry (LDI-TOF-MS) data were obtained using a Shimadzu AXIMA Performance MALDI-TOF instrument in positive detection modes with an applied voltage of $25 \mathrm{kV}$ between the target and the aperture of the time-of-flight analyzer. Elemental analyses were performed on a flash EA 1112 spectrometer.

Synthesis of 5-(4-(di([1,1'-biphenyl]-4-yl)amino)phenyl)acenaphthylene-1,2-dione (4-(di([1,1'-biphenyl]-4-yl)amino)phenyl)boronic acid $(2.11 \quad \mathrm{~g}, 4.8 \mathrm{mmol}), 5^{-}$ Bromoacenaphthylene-1,2-dione $(1.04 \mathrm{~g}, 4 \mathrm{mmol}), \mathrm{K}_{2} \mathrm{CO}_{3}(1.66 \mathrm{~g}, 12 \mathrm{mmol})$ and $\mathrm{Pd}\left(\mathrm{PPh}_{3}\right)_{4}$ $(0.46 \mathrm{~g}, 0.4 \mathrm{mmol})$ were dissolved in a $100 \mathrm{~mL}$ round-bottle flash with $30 \mathrm{~mL}$ dioxane and 10 $\mathrm{mL}$ deionized water under an argon atmosphere. Then the mixture was stirred under reflux with the temperature of $100^{\circ} \mathrm{C}$ for 24 hours. After cooling to room temperature, the mixture was poured into $50 \mathrm{~mL}$ water and extracted with dichloromethane for three times. The obtained organic layer was washed with water and then dried over $\mathrm{MgSO}_{4}$ and concentrated by rotary evaporator. The crude product was purified by column chromatography on silica gel (hexane/dichloromethane $=1: 5, \mathrm{v} / \mathrm{v})$ to give $5-\left(4-\left(\mathrm{di}\left(\left[1,1^{\prime}-\right.\right.\right.\right.$ biphenyl $]-4^{-}$ 
yl)amino)phenyl)acenaphthylene-1, 2-dione as deep-red solid. Yield: $57 \%$. ${ }^{1} \mathrm{H}$ NMR $(600 \mathrm{MHz}$, $\left.\mathrm{CDCl}_{3}\right): \delta=8.50(\mathrm{dd}, J=8.5,0.8 \mathrm{~Hz}, 1 \mathrm{H}), 8.17(\mathrm{~d}, J=7.3 \mathrm{~Hz}, 1 \mathrm{H}), 8.12(\mathrm{dd}, J=7.0,0.7 \mathrm{~Hz}, 1 \mathrm{H})$, 7.86-7.80 (m, 2H), 7.63-7.59 (m, 4H), 7.59-7.56 (m, 4H), 7.54-7.51 (m, $2 \mathrm{H}), 7.47-7.42(\mathrm{~m}, 4 \mathrm{H})$, 7.36-7.29 (m, 8H). HRMS (MALDI-TOF): calcd for $\mathrm{C}_{42} \mathrm{H}_{27} \mathrm{NO}_{2}[M]^{+}: 577.2042$, found: 577.2330 .

Synthesis of 3-(4-(di([1,1'-biphenyl]-4-yl)amino)phenyl)acenaphtho[1,2-b]pyrazine-8,9dicarbonitrile (DPTPAAP)

5-(4-(di([1,1'-biphenyl]-4-yl)amino)phenyl)acenaphthylene-1,2-dione (1.5 g, $2.6 \mathrm{mmol})$ and diaminomaleonitrile $(0.42 \mathrm{~g}, 3.9 \mathrm{mmol}$ ) were dissolved in a $500 \mathrm{~mL}$ round-bottle flash with $300 \mathrm{~mL}$ glacial acetic acid. Under argon atmosphere, the mixture was stirred under reflux with the temperature of $130^{\circ} \mathrm{C}$ for 24 hours. After cooling to room temperature, the mixture was filtered off and washed with acetone, ethanol, water and then dried under vacuum. The crude product was purified by column chromatography on silica gel using hexane/dichloromethane $(2: 1, v / v)$ as eluent to give dpTPAAP as dark purple solid. Yield: $84 \%$. This solid was further purified through temperature gradient vacuum sublimation under the temperature of $300-320^{\circ} \mathrm{C}$ and atmospheric pressure of $7 \times 10^{-4} \mathrm{~Pa}$ gave dpTPAAP as a black

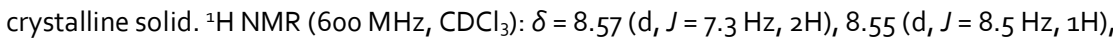
8.00-7.95 (m, 2H), 7.64-7.56 (m, 10H), $7.46(\mathrm{t}, J=7.7 \mathrm{~Hz}, 4 \mathrm{H}), 7.40-7.31(\mathrm{~m}, 8 \mathrm{H}) .{ }^{13} \mathrm{C}$ NMR $(150$ $\left.\mathrm{MHz}, \mathrm{CDCl}_{3}\right): \delta=154.39,154.06,148.47,146.71,146.34,140.41,136.63,136.01,132.23,131.48$, $131.28,129.92,129.66,129.37,129.19,128.84,128.68,128.17,127.15,126.94,126.77,126.32$, $126.06,125.25,122.97,114.32,114.28$. HRMS (MALDI-TOF): calcd for $\mathrm{C}_{46} \mathrm{H}_{27} \mathrm{~N}_{5}[M]^{+}$: 649.2266, found: 649.2368. Elemental analysis calcd for $\mathrm{C}_{46} 6 \mathrm{H}_{27} \mathrm{~N}_{5}: \mathrm{C}, 85.03 ; \mathrm{H}, 4.19 ; \mathrm{N}, 10.78$. Found: $\mathrm{C}, 84.85 ; \mathrm{H}, 4.38 ; \mathrm{N}, 10.67$.

Synthesis of 3-(4-(di([1,1'-biphenyl]-4-yl)amino)phenyl)acenaphtho[1,2-b]pyrazino[2,3e]pyrazine-9,10-dicarbonitrile (dPTPAAZ)

5-(4-(di([1,1'-biphenyl]-4-yl)amino)phenyl)acenaphthylene-1,2-dione $(1.5 \mathrm{~g}, 2.6 \mathrm{mmol})$ and 5,6-diaminopyrazine-2,3-dicarbonitrile $(0.62 \mathrm{~g}, 3.9 \mathrm{mmol}$ ) were dissolved in a $500 \mathrm{~mL}$ roundbottle flash with $300 \mathrm{~mL}$ glacial acetic acid. Under argon atmosphere, the mixture was stirred under reflux with the temperature of $130^{\circ} \mathrm{C}$ for 24 hours. After cooling to room temperature, the mixture was filtered off and washed with acetone, ethanol, water and then dried under vacuum. The crude product was purified by column chromatography on silica gel using hexane/dichloromethane $(7: 1, v / v)$ as elvent to give dpTPAAZ as black solid. Yield: $81 \%$. This solid was further purified through temperature gradient vacuum sublimation under the temperature of $360-380{ }^{\circ} \mathrm{C}$ and atmospheric pressure of $7 \times 10^{-4} \mathrm{~Pa}$ gave dpTPAAZ as a black solid. ${ }^{1} \mathrm{H}$ NMR $\left(600 \mathrm{MHz}, \mathrm{CD}_{2} \mathrm{Cl}_{2}\right): \delta=8.77-8.73(\mathrm{~m}, 2 \mathrm{H}), 8.64(\mathrm{~d}, J=8.4 \mathrm{~Hz}, 1 \mathrm{H}), 8.08-8.04(\mathrm{~m}$, $2 \mathrm{H}), 7.68-7.58(\mathrm{~m}, 10 \mathrm{H}), 7.46(\mathrm{t}, J=7.7 \mathrm{~Hz}, 4 \mathrm{H}), 7.40-7.31(\mathrm{~m}, 8 \mathrm{H}) .{ }^{13} \mathrm{C}$ NMR of dpTPAAZ was not obtained because of its low solubility in $\mathrm{CDCl}_{3}$. HRMS (MALDI-TOF): calcd for $\mathrm{C}_{48} \mathrm{H}_{28} \mathrm{~N}_{7}{ }^{+}$

$[\mathrm{M}+\mathrm{H}]^{+}:$701.2401, found: 702.1460. Elemental analysis calcd for $\mathrm{C}_{48} \mathrm{H}_{27} \mathrm{~N}_{7}: \mathrm{C}, 82.15 ; \mathrm{H}, 3.88$;

$\mathrm{N}, 13.97$. Found: $\mathrm{C}, 82.06 ; \mathrm{H}, 4.00 ; \mathrm{N}, 13.89$.

\section{Single-crystal Structure}

The single crystal of dpTPAAP was obtained in the process of vacuum train sublimation. The low temperature (104.6K) single-crystals $X$-ray experiments were performed on a Rigaku RAXIS-SPIDER IP diffractometer with graphite-monochromatized MoK $\alpha$ radiation $(\lambda=0.71073 \AA)$. Data collection and reduction, cell refinement, and experiential absorption correction for all compounds were performed with the Rigaku RAPID AUTO software package (Rigaku, 1998, Version 2.30). CCDC 1975023 contain the supplementary crystallographic data for this paper. These data can be obtained free of charge from The Cambridge Crystallographic Data Centre via www.ccdc.cam.ac.uk/data_request/cif.

\section{Thermal Properties}

Thermogravimetric analyses (TGAs) were performed on a STA $409 \mathrm{PC}$ thermogravimeter by measuring the weight loss while heating at a rate of $10^{\circ} \mathrm{C}$ min-1 under nitrogen.

\section{Electron Paramagnetic Resonance}

The EPR measurements were performed on the X-band EPR spectrometer (JEOL, JES FA200) at room temperature. The EPR measurement under photoexcitation were carried out using a broadband Xe lamp. 
Photophysical Measurement

The $\Phi_{\mathrm{PL}}$ was measured using an absolute photoluminescence quantum yield measurement system (Hamamatsu C9920-03G) with an excitation wavelength of $390 \mathrm{~nm}$. Absorption spectra of solutions and films were obtained on an Agilent 8453 UV-vis/NIR spectrophotometer and the absorption spectrum of single crystal was obtained on a Hitachi U-3900 UV-vis/NIR spectrophotometer. Emission spectra were collected on an Edinburgh FLS-920 fluorospectrophotometer equipped with a R928P photomultiplier tube with a spectral response range from 200 to $850 \mathrm{~nm}$ and a Hamamatsu R5509 photomultiplier tube with response range from 300 to $1700 \mathrm{~nm}$. The fluorescence spectra and phosphorescence spectra used for determining the $S_{1}$ and $T_{1}$ values were collected on an Edinburgh FLS-920 fluorospectrophotometer with an excitation wavelength of $490 \mathrm{~nm}$. The fluorescence spectra of films were obtained by steady-state photoluminescence measurements at room temperature without time gating, and the phosphorescence spectra of films were recorded by time-gated photoluminescence measurements (delayed by $5 \mathrm{~ms}$ ) at $77 \mathrm{~K}$ with a integration time of $5 \mathrm{~ms}$. Transient PL measurements were carried out using a transient spectrometer (Edinburg FLS-920). The prompt parts of transient spectra were collected with a picosecond pulsed Laser (EPL-510, $\lambda_{\text {ex }}=505.4 \mathrm{~nm}$ ), while the delayed parts of transient spectra were collected with a $\mu \mathrm{F} 2$ microflash lamp $\left(\lambda_{\mathrm{ex}}=505 \mathrm{~nm}\right)$. The $1 \times 10^{-5} \mathrm{M}$ solution was used for $\mathrm{PL}$ spectra measurement. Small-molecule organic films for optical measurements were fabricated by thermal evaporation under high vacuum $\left(10^{-4} \mathrm{~Pa}\right)$ onto clean quartz substrates.

\section{Theoretical Calculation}

Calculations on the ground and excited electronic states were performed in Gaussian 16 with density functional theory (DFT) and time-dependent DFT (TDDFT) using an optimal tuning version of $\omega B$ B7XD long-range corrected functional $(\omega=0.1264)$ with def2-SVP basis set in gas phase. ${ }^{32,45-46}$ The input files for the hole/electron excitation analysis were prepared by printing orbital pairs with a configuration coefficient $>0.001$ using keyword with $\operatorname{IOP}(9 / 40=$ 3). The diabatization of the electronic states and analysis were performed using $\mathrm{Q}$-Chem and Multiwfn.

\section{Device Fabrication and Measurements}

The devices were fabricated by thermal evaporation under high vacuum (ca. $4 \times 10-4 \mathrm{~Pa}$ ) onto ITO-coated glass substrates. The substrates were carefully cleaned and treated with UV ozone for 10 min before vacuum thermal deposition. All OLED devices were measured in ambient. The voltage, current, luminance and radiant flux characteristics were measured using a Keithley 2400 source meter and an absolute EQE measurement system (Hamamatsu C9920-12 equipped with Hamamatsu PMA-12 Photonic multichannel analyzer (10027-02).

\section{SUPPLEMENTAL INFORMATION}

Supplemental Information includes Supplemental Experimental Procedures, seventeen figures, and four tables, and can be found with this article online.

\section{ACKNOWLEDGMENTS}

This work was supported by the National Key R\&D Program of China (Grant No. 2016YFB0401003, No. 2017YFA0204501), National Natural Science Foundation of China (Grant No. 21788102, No. 51773109), National Postdoctoral Program for Innovative Talents (Grant No. BX20180159), and the Project funded by China Postdoctoral Science Foundation (2019M660606).

\section{AUTHOR CONTRIBUTIONS}

J.Xue, J.Xu and Q.L. designed, synthesized and characterized the compounds. J.Xue and J.Xu measured physicochemical properties of materials. J.R. developed the theoretical approach. J.R. and J.Xue performed the computational experiments. Z.S., Q.O. and R.W. participated in the theoretical investigations. J.Xue, J.R. and J.Q. participated in data analysis and wrote the paper. J.Q. supervised the whole work. All authors discussed the results and commented on the manuscript.

\section{DECLARATION OF INTERESTS}

The authors declare no competing interests. 


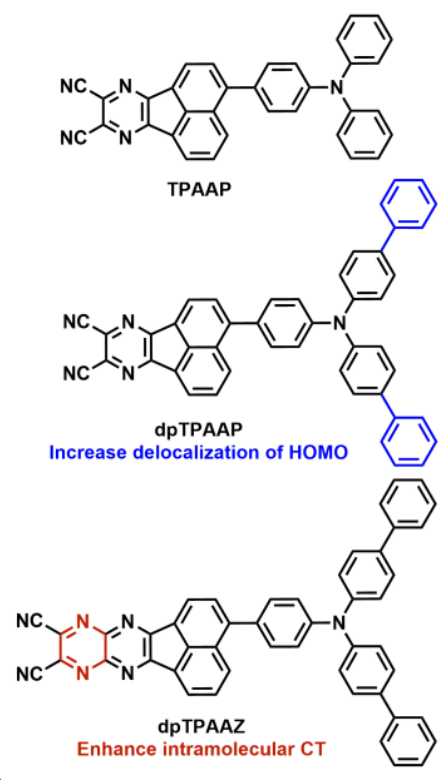

Figure 1. Molecular design

Design strategies for organic NIR emitters and chemical structures of dpTPAAP and dPTPAAZ.
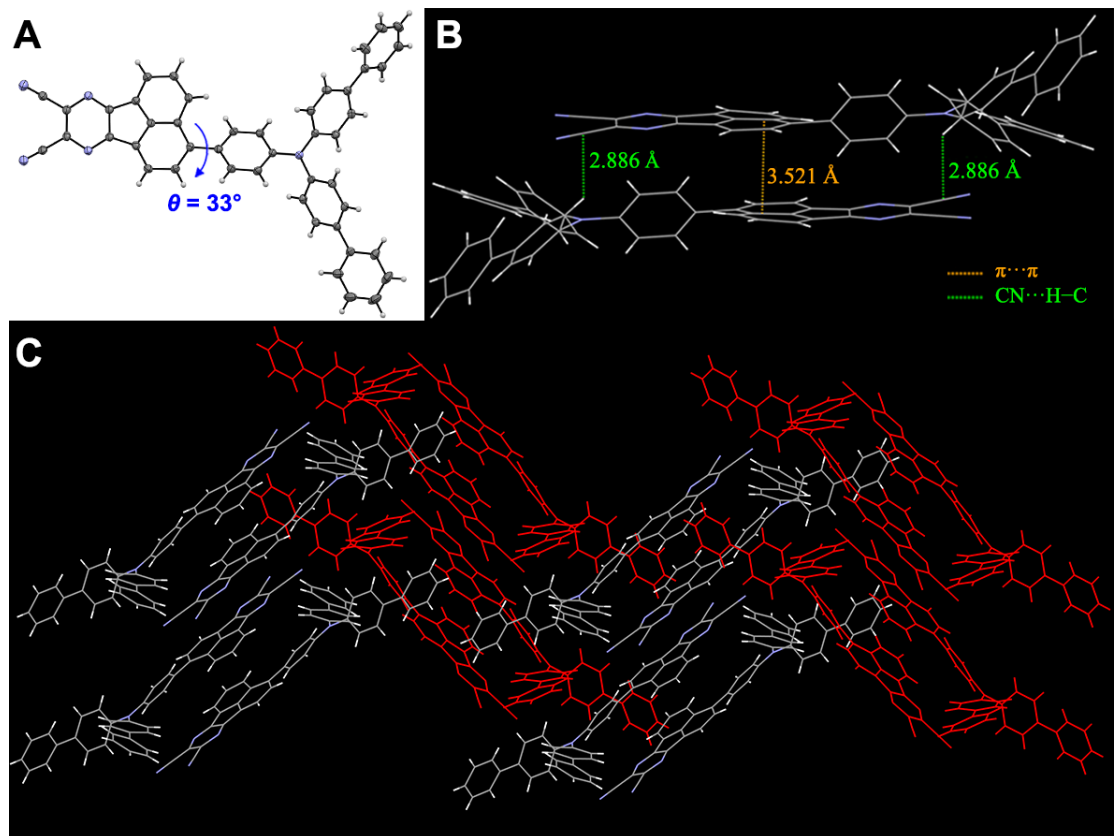

Figure 2. Single-Crystal Structure

(A) Single-crystal structure of dpTPAAP with thermal ellipsoids plotted at 50\% probability level.

(B) Packing motifs of aggregated dimers.

(C) Packing motifs of dpTPAAP in single crystals. 

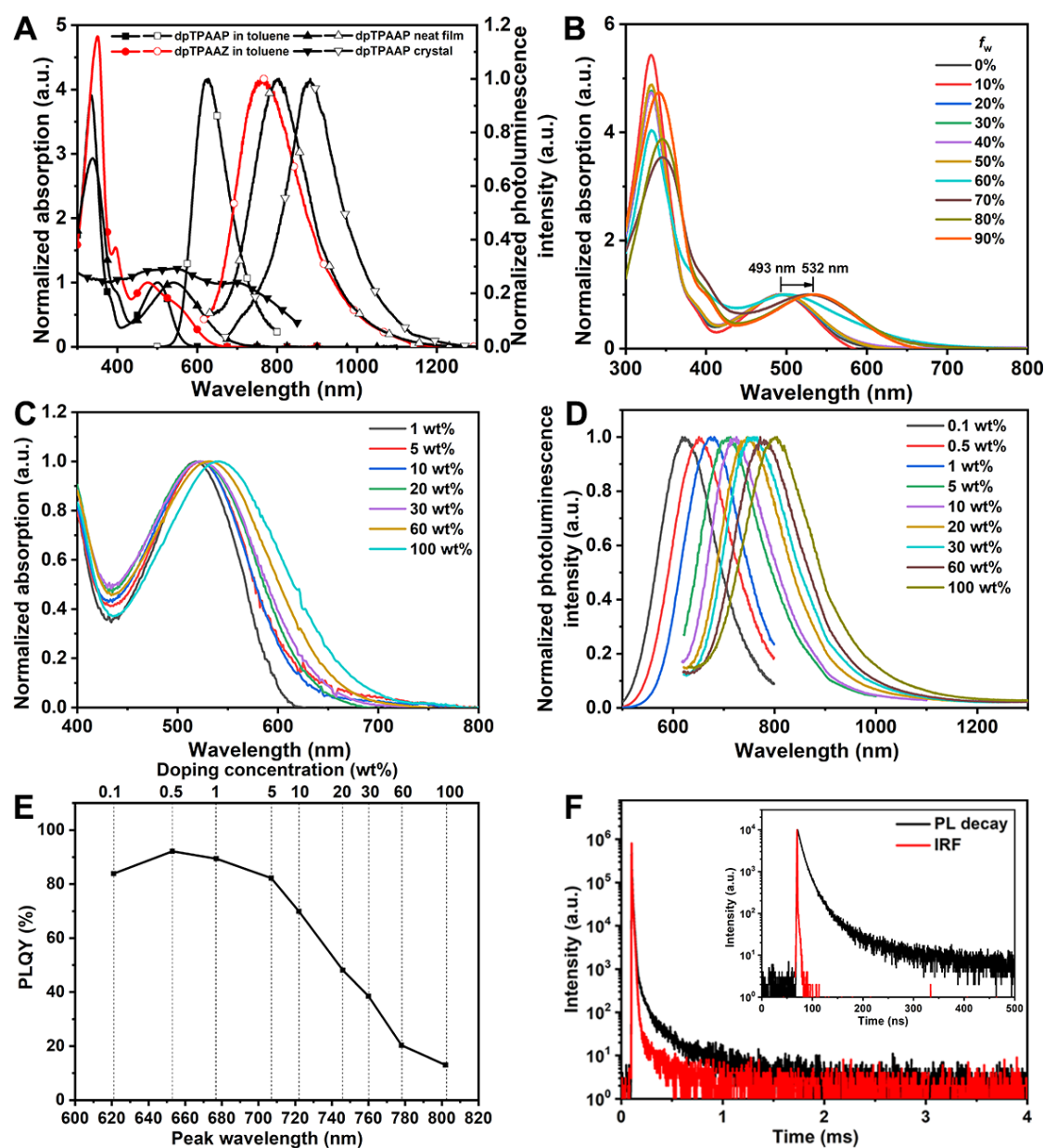

Figure 3. Photophysical Properties

(A) Absorption (solid symbol) and PL (open symbol) spectra of dpTPAAP and dpTPAAZ in toluene $\left(1 \times 10^{-5} \mathrm{~mol} \mathrm{~L}^{-1}\right)$, dpTPAAP in neat film and single crystal.

(B) Absorption spectra of dpTPAAP in THF/water mixtures $\left(1 \times 10^{-5} \mathrm{~mol} \mathrm{~L}^{-1}\right)$ with different water fractions $\left(f_{\mathrm{w}}\right)$.

(C-D) Absorption spectra (C), PL spectra (D) and PLOY (E) of dpTPAAP in solid films with TPBi as host at various doping concentrations at room temperature.

(F) Transient PL decay curves of $30 \mathrm{wt} \%$ dpTPAAP:TPBi film. Inset: the prompt decay curves in nanosecond scale.
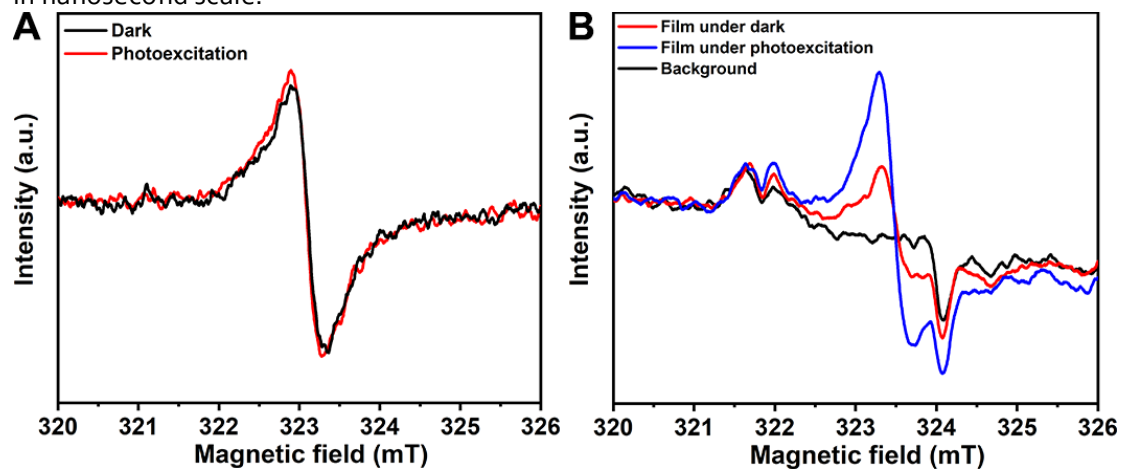

Figure 4. Electron Paramagnetic Resonance

(A and B) EPR signals of dpTPAAP single crystals (A) and neat film (B) under dark condition and photoexcitation. 


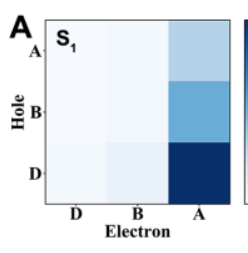

Hole

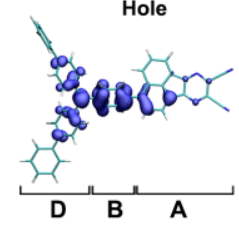

Electron

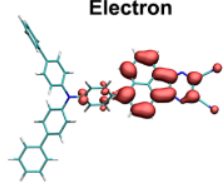

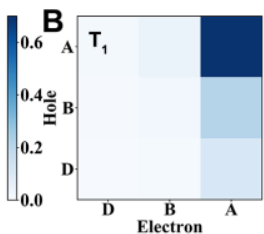

Hole
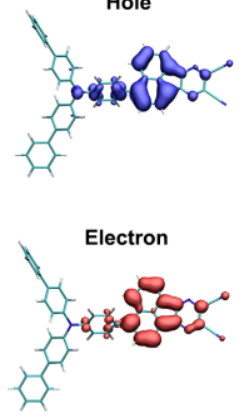

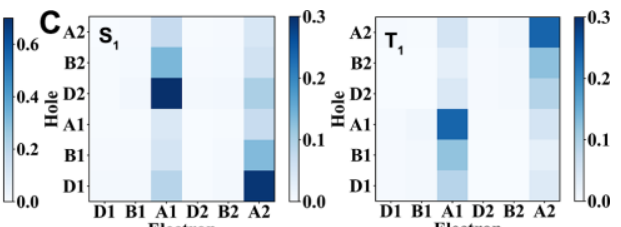

D ${ }_{\mathrm{xCT}} \quad \mathbf{x C T}_{2} \quad \mathrm{iCT}, \quad \mathrm{iCT}_{2}$ Electron

$\left[\begin{array}{cccc}\mathbf{x} \mathbf{C T}_{1} & \mathbf{x C T}_{2} & \mathbf{i C T} & { }^{\mathbf{i C T}}{ }_{2} \\ 2385 & -3 & 19 & -103 \\ -3 & -385 & -103 & 19\end{array}\right]$ $\mathrm{H}=\left[\begin{array}{cccc}2385 & -3 & 19 & -103 \\ -3 & 2385 & -103 & 19 \\ 19 & -103 & 2541 & 26 \\ -103 & 19 & 26 & 2541\end{array}\right] \mathrm{meV}$ Hole Electron

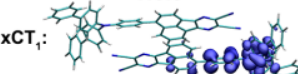

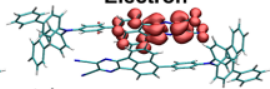

$\mathrm{xCT}_{2}$ :

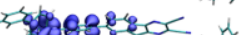

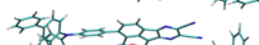

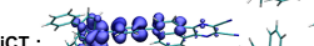

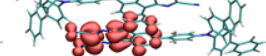

i.

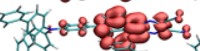

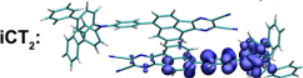
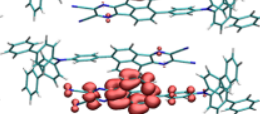

Figure 5. Quantum chemical calculations

(A and B) Fragment-based TDM (D/B/A denotes donor/bridge/acceptor) and attachment(electron)/detachment(hole) density (isovalue $=0.001$ ) for $(A) S_{1}$ and $(B) T_{1}$ state of dpTPAAP monomer.

(C) TDM for $S_{1} / T_{1}$ states of dpTPAAP dimer 1.

(D) The diabatic Hamiltonian and electron/hole density of the lowest 4 diabatic states ( $2 \times C T$ and $2 \mathrm{iCT}$ ) of dPTPAAP dimer 1.

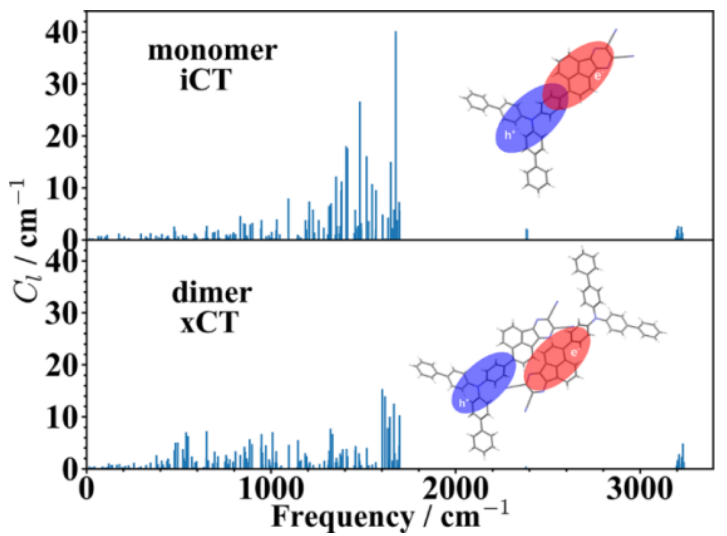

Figure 6. Nonadiabatic coupling elements

The prefactor $C_{l}$ of $k_{\mathrm{nr}}$ in Eq. (1) for dpTPAAP monomer and dimer 1 at their optimized S。 structures. 

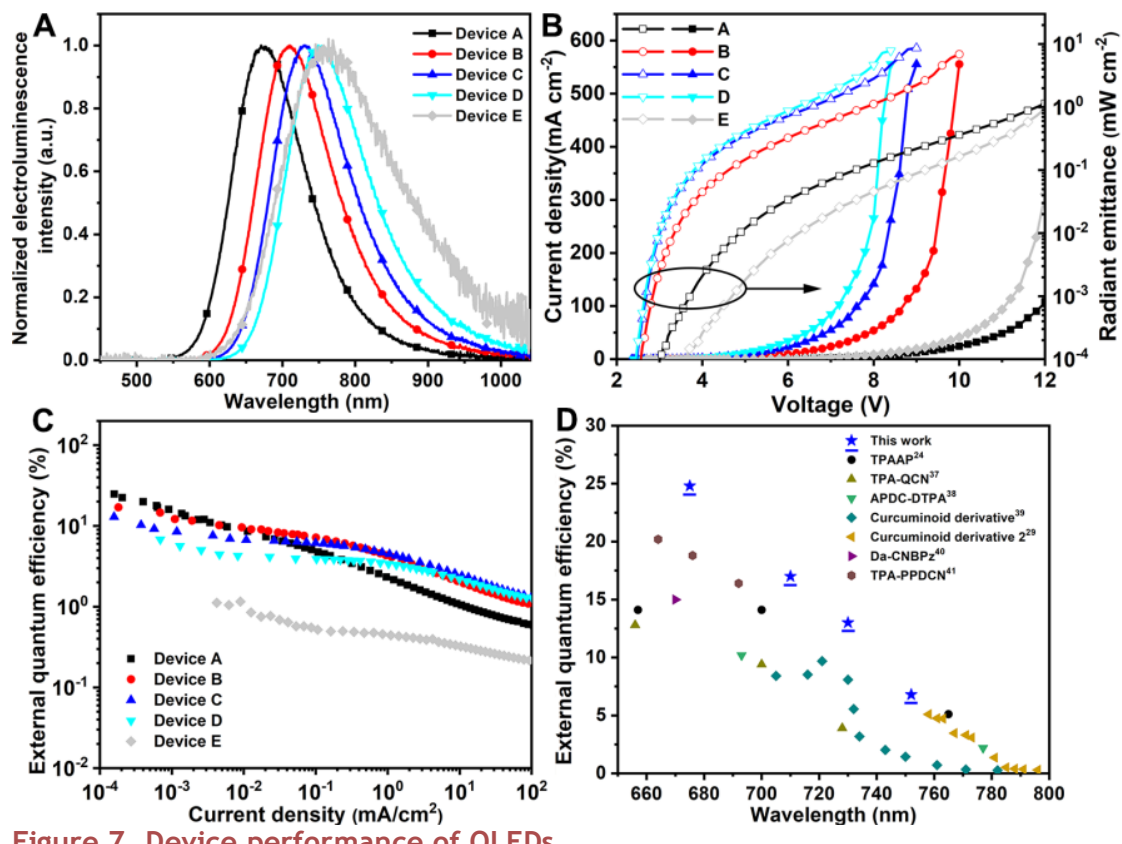

Figure 7. Device performance of OLEDs

(A) Electroluminescence spectra recorded th the voltage of $1 \mathrm{~mA} \mathrm{~cm}^{-2}$ for devices A, B, C, D and $\mathrm{E}$.

(B) Current density-voltage-radiant emittance characteristics for devices A, B, C, D and E.

(C) EQE as a function of current density.

(D) Maximum EQE summary of the representative deep-red and NIR OLEDs based on TADF emitters with emission peaks from $650 \mathrm{~nm}$ to $800 \mathrm{~nm}$.

Table 1. Electroluminescence characteristics of the devices

\begin{tabular}{ccccc}
\hline Device & $\begin{array}{c}V_{\mathrm{On}^{\mathrm{a}}} \\
(\mathrm{V})\end{array}$ & $\begin{array}{c}\lambda_{\mathrm{EL}, \mathrm{max}^{\mathrm{b}}} \\
(\mathrm{nm})\end{array}$ & $\operatorname{EQE}^{\mathrm{C}}(\%)$ & $\begin{array}{c}\text { Radiant } \\
\text { emittance } \\
\left(\mathrm{W} \mathrm{m} \mathrm{m}^{-2}\right)\end{array}$ \\
\hline A & 3.0 & 675 & $24.8 / 2.3 / 1.1$ & $11.40^{\mathrm{d}}$ \\
B & 2.6 & 710 & $17.0 / 4.3 / 2.0$ & $69.06^{\mathrm{e}}$ \\
C & 2.5 & 730 & $13.0 / 4.6 / 2.5$ & $86.02^{\mathrm{f}}$ \\
D & 2.5 & 752 & $6.8 / 3.4 / 2.2$ & $78.36^{\mathrm{g}}$ \\
E & 3.6 & 757 & $1.2 / 0.4 / 0.3$ & $9.44^{\mathrm{d}}$ \\
\hline
\end{tabular}

${ }^{\mathrm{a}} \mathrm{Von}_{\mathrm{on}}$ is the onset voltage obtained at $1 \mathrm{~mW} \mathrm{~m}^{-2}$.

${ }^{b}$ Recorded at $1 \mathrm{~mA} \mathrm{~cm}^{-2}$.

'EQE: maximum, value at $1 \mathrm{~mA} \mathrm{~cm}^{-2}$ and value at $10 \mathrm{~mA} \mathrm{~cm}^{-2}$, respectively;

dRecorded at $12 \mathrm{~V}$.

eRecorded at $10 \mathrm{~V}$.

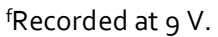

gRecorded at $8.4 \mathrm{~V}$.

\section{REFERENCES}

1. Bünzli, J.-C. G.; Eliseeva, S. V. (2010). Lanthanide NIR luminescence for telecommunications, bioanalyses and solar energy conversion. $J$. Rare Earths 28, 824-842. 10.1016/S1002-0721(09)60208-8.

2. Kenry; Duan, Y.; Liu, B. (2018). Recent Advances of Optical Imaging in the Second Near-
Infrared Window. Adv. Mater. 30 1802394. 10.1002/adma.201802394.

3. Oian, G.; Wang, Z. Y. (2010). NearInfrared Organic Compounds and Emerging Applications. Chem. Asian J. 5, 1006-1029. 10.1002/asia.200900596.

4. Zampetti, A.; Minotto, A.; Cacialli, F. (2019). Near-Infrared (NIR)
Organic Light-Emitting Diodes (OLEDs): Challenges and Opportunities. Adv. Funct. Mater. 29, 1807623 . 10.1002/adfm.201807623.

5. Chen, S.; Weitemier, A. Z.; Zeng, $X_{\text {.; }}$ He, L.; Wang, X.; Tao, Y.; Huang, A. J. Y.; Hashimotodani, $Y$.; Kano, M.; Iwasaki, H., et al. (2018). Near-infrared deep brain 
stimulation via upconversion nanoparticle-mediated optogenetics. Science 359, 679 10.1126/science.aaq1144.

6. Khan, Y.; Han, D.; Pierre, A.; Ting, J.; Wang, X.; Lochner, C. M.; Bovo, G.; Yaacobi-Gross, N.; Newsome, C.; Wilson, R., et al. (2018). A flexible organic reflectance oximeter array. Proc. Natl. Acad. Sci. U.S. A. 115, E11015. 10.1073/pnas.1813053115.

7. Takahiko, Y.; Hajime, N.; Shigeo, H.; Toru, H.; Chihaya, A. (2017). Near-infrared organic lightemitting diodes for biosensing with high operating stability. Appl. Phys. Express 10, 074101.

8. Jeon, Y.; Choi, H.-R.; Kwon, J. H.i Choi, S.; Nam, K. M.; Park, K.-C.; Choi, K. C. (2019). Sandwichstructure transferable free-form OLEDs for wearable and disposable skin wound photomedicine. Light: Sci. Appl. 8, 114. 10.1038/s41377-019-0221-3.

9. Khan, Y.; Han, D.; Ting, J.; Ahmed, M.; Nagisetty, R.; Arias, A. C. (2019). Organic Multi-Channel Optoelectronic Sensors for Wearable Health Monitoring. IEEE Access 7, 128114-128124. 10.1109/ACCESS.2019.2939798.

10. Siebrand, W. (1967). Radiationless Transitions in Polyatomic Molecules. I. Calculation of Franck-Condon Factors. J. Chem. Phys. 46, 440-447. 10.1063/1.1840685.

11. Englman, R.; Jortner, J. (1970). The energy gap law for radiationless transitions in large molecules. Mol. Phys. 18, 145-164. 10.1080/00268977000100171.

12. Yao, L.; Zhang, S.; Wang, R.; Li, W.; Shen, F.; Yang, B.; Ma, Y. (2014). Highly Efficient NearInfrared Organic Light-Emitting Diode Based on a Butterfly-Shaped Donor-Acceptor Chromophore with Strong Solid-State Fluorescence and a Large Proportion of Radiative Excitons. Angew. Chem. Int. Ed. 53, 21192123. 10.1002/anie.201308486.

13. Wang, S.; Yan, X.; Cheng, Z.; Zhang, H.; Liu, Y.; Wang, Y. (2015). Highly Efficient Near-Infrared Delayed Fluorescence Organic Light Emitting Diodes Using a Phenanthrene-Based Charge-
Transfer Compound. Angew. Chem. Int. Ed. 54, 13068-13072. 10.1002/anie.201506687.

14. Peng, Q.; Obolda, A.; Zhang, M.; Li, F. (2015). Organic LightEmitting Diodes Using a Neutral $\pi$ Radical as Emitter: The Emission from a Doublet. Angew. Chem. Int. Ed. 54, 7091-7095. 10.1002/anie.201500242.

15. Ai, X.; Evans, E. W.; Dong, S.; Gillett, A. J.; Guo, H.; Chen, Y.; Hele, T. J. H.; Friend, R. H.; Li, F. (2018). Efficient radical-based light-emitting diodes with doublet emission. Nature 563, 536-540. 10.1038/s41586-018-0695-9.

16. Deng, C.; Niu, Y.; Peng, Q.; Qin, A.; Shuai, Z.; Tang, B. Z. (2011). Theoretical study of radiative and non-radiative decay processes in pyrazine derivatives. J. Chem. Phys. 135, 014304. 10.1063/1.3606579.

17. Metcalfe, J.; Phillips, D. (1976). Photophysical processes in fluorinated acetones. J. Chem. Soc., Faraday Trans. 2 72, 1574-1583. 10.1039/F29767201574.

18. Siebrand, W.; Williams, D. F. (1968). Radiationless Transitions in Polyatomic Molecules. III. Anharmonicity, Isotope Effects, and Singlet-to-Ground-State Transitions in Aromatic Hydrocarbons. J. Chem. Phys. 49, 1860-1871. 10.1063/1.1670318.

19. Yang, J.; Chi, Z.; Zhu, W.; Tang, B. Z.; Li, Z. (2019). Aggregationinduced emission: a coming-of-age ceremony at the age of eighteen. Sci. China Chem. 62, 1090-1098. 10.1007/s11426-019-9512-X.

20. Wu, Q.; Deng, C.; Peng, Q.; Niu, Y.; Shuai, Z. (2012). Quantum chemical insights into the aggregation induced emission phenomena: A OM/MM study for pyrazine derivatives. J. Comput. Chem. 33, 1862-1869. 10.1002/jcc.23019.

21. Chen, W.-C.; Chou, P.-T.; Cheng, Y.-C. (2019). Low Internal Reorganization Energy of the Metal-Metal-to-Ligand Charge Transfer Emission in Dimeric Pt(II) Complexes. J. Phys. Chem. C 123, 10225-10236. 10.1021/acs.jpcc. gboo224.

22. Wei, Y.-C.; Wang, S. F.; Hu, Y.; Liao, L.-S.; Chen, D.-G.; Chang, K.H.; Wang, C.-W.; Liu, S.-H.; Chan,
W.-H.; Liao, J.-L., et al. (2020). Overcoming the energy gap law in near-infrared OLEDs by excitonvibration decoupling. Nat. Photonics 14, 570-577. 10.1038/s41566-020-0653-6.

23. Chernyak, V.; Mukamel, S. (2000). Density-matrix representation of nonadiabatic couplings in timedependent density functional (TDDFT) theories. J. Chem. Phys. 112, 3572-3579. 10.1063/1.480511.

24. Xue, J.; Liang, Q.; Wang, R.; Hou, J.; Li, W.; Peng, Q.; Shuai, Z.; Qiao, J. (2019). Highly Efficient Thermally Activated Delayed Fluorescence via J-Aggregates with Strong Intermolecular Charge Transfer. Adv. Mater. 31, 1808242. 10.1002/adma.201808242.

25. Hirata, S.; Sakai, Y.; Masui, K.; Tanaka, H.; Lee, S. Y.; Nomura, H.; Nakamura, N.; Yasumatsu, M.; Nakanotani, H.; Zhang, Q., et al. (2014). Highly efficient blue electroluminescence based on thermally activated delayed fluorescence. Nat. Mater. 14, 330. 10.1038/nmat4154.

26. Zhang, Q.; Kuwabara, H.; Potscavage, W. J.; Huang, S.; Hatae, Y.; Shibata, T.; Adachi, C. (2014). Anthraquinone-Based Intramolecular Charge-Transfer Compounds: Computational Molecular Design, Thermally Activated Delayed Fluorescence, and Highly Efficient Red Electroluminescence. J. Am. Chem. Soc. 136, 18070-18081. 10.1021/ja510144h.

27. Chen, W.-C.; Huang, B.; Ni, S.-F.; Xiong, Y.; Rogach, A. L.; Wan, Y.; Shen, D.; Yuan, Y.; Chen, J.-X.; Lo, M.-F., et al. (2019). Deep-

Red/Near-Infrared Electroluminescence from SingleComponent Charge-Transfer Complex via Thermally Activated Delayed Fluorescence Channel. Adv. Funct. Mater. 29, 1903112. 10.1002/adfm.201903112.

28. Liang, Q.; Xu, J.; Xue, J.; Qiao, J. (2020). Near-infrared-Il thermally activated delayed fluorescence organic light-emitting diodes. Chem. Commun. 56, 8988-8991. 10.1039/DoCC02695F.

29. Ye, H.; Kim, D. H.; Chen, X.; Sandanayaka, A. S. D.; Kim, J. U.; Zaborova, E.; Canard, G.; Tsuchiya, 
Y.; Choi, E. Y.; Wu, J. W., et al. (2018). Near-Infrared

Electroluminescence and Low Threshold Amplified Spontaneous Emission above $800 \mathrm{~nm}$ from a Thermally Activated Delayed Fluorescent Emitter. Chem. Mater. 30, 6702-6710.

10.1021/acs.chemmater.8bo2247.

30. Madigan, C. F.; Bulović, V. (2003).

Solid State Solvation in

Amorphous Organic Thin Films.

Phys. Rev. Lett. 91, 247403.

31. Wang, M.; Huang, Y.-H.; Lin, K.-S.;

Yeh, T.-H.; Duan, J.; Ko, T.-Y.; Liu,

S.-W.; Wong, K.-T.; Hu, B. (2019).

Revealing the Cooperative

Relationship between Spin,

Energy, and Polarization

Parameters toward Developing

High-Efficiency Exciplex Light-

Emitting Diodes. Adv. Mater.

1904114 .

10.1002/adma.201904114.

32. Kronik, L.; Kümmel, S. (2018).

Dielectric Screening Meets

Optimally Tuned Density

Functionals. Adv. Mater. 30 , 1706560.

10.1002/adma.201706560.

33. Shao, Y.; Gan, Z.; Epifanovsky, E.; Gilbert, A. T. B.; Wormit, M.; Kussmann, J.; Lange, A. W.; Behn, A.; Deng, J.; Feng, X., et al. (2015). Advances in molecular quantum chemistry contained in the QChem 4 program package. Mol. Phys. 113, 184-215. 10.1080/00268976.2014.952696.

34. Lu, T.; Chen, F. (2012). Multiwfn: A multifunctional wavefunction analyzer. J. Comput. Chem. 33, 580592. 10.1002/jcc.22885.

35. Biswas, S.; Pramanik, A.; Pal, S.; Sarkar, P. (2017). A Theoretical Perspective on the Photovoltaic Performance of S,N-Heteroacenes: An Even-Odd Effect on the Charge
Separation Dynamics. J. Phys.

Chem. C 121, 2574-2587.

10.1021/acs.jpcc.6b11471.

36. Chen, X.-K.; Ravva, M. K.; Li, H.;

Ryno, S. M.; Brédas, J.-L. (2016).

Effect of Molecular Packing and

Charge Delocalization on the

Nonradiative Recombination of

Charge-Transfer States in Organic

Solar Cells. Adv. Energy Mater. 6,

1601325 .

10.1002/aenm.201601325.

37. Li, C.; Duan, R.; Liang, B.; Han, G.; Wang, S.; Ye, K.; Liu, Y.; Yi, Y.; Wang, Y. (2017). Deep-Red to Near-Infrared Thermally Activated Delayed Fluorescence in Organic Solid Films and Electroluminescent Devices. Angew. Chem. Int. Ed. 56, 11525-11529. 10.1002/anie.201706464.

38. Yuan, Y.; Hu, Y.; Zhang, Y.-X.; Lin, J.-D.; Wang, Y.-K.; Jiang, Z.-O.; Liao, L.-S.; Lee, S.-T. (2017). Over 10\% EQE Near-Infrared Electroluminescence Based on a Thermally Activated Delayed Fluorescence Emitter. Adv. Funct. Mater. 27, 1700986.

10.1002/adfm.201700986.

39. Kim, D.-H.; D'Aléo, A.; Chen, X.-K.; Sandanayaka, A. D. S.; Yao, D.; Zhao, L.; Komino, T.; Zaborova, E.; Canard, G.; Tsuchiya, Y., et al. (2018). High-efficiency electroluminescence and amplified spontaneous emission from a thermally activated delayed fluorescent near-infrared emitter. Nat. Photonics 12, 98-104. 10.1038/s41566-017-0087-y.

40. Furue, R.; Matsuo, K.; Ashikari, Y.; Ooka, H.; Amanokura, N.; Yasuda, T. (2018). Highly Efficient RedOrange Delayed Fluorescence Emitters Based on Strong $\pi-$ Accepting Dibenzophenazine and Dibenzoquinoxaline Cores: toward a Rational Pure-Red OLED Design. Adv. Opt. Mater. 6, 1701147. 10.1002/adom.201701147.

41. Yang, T.; Liang, B.; Cheng, Z.; Li, C.; LU, G.; Wang, Y. (2019). Construction of Efficient DeepRed/Near-Infrared Emitter Based on a Large $\pi$-Conjugated Acceptor and Delayed Fluorescence OLEDs with External Quantum Efficiency of over $20 \%$. J. Phys. Chem. C 123, 18585-18592.

10.1021/acs.jpcc. gbo5875.

42. Li, C.; Duan, L.; Li, H.; Qiu, Y.

(2013). Percolative charge transport in a co-evaporated organic molecular mixture. Org. Electron. 14, 3312-3317. 10.1016/j.orgel.2013.09.039.

43. Murawski, C.; Leo, K.; Gather, M. C. (2013). Efficiency Roll-Off in Organic Light-Emitting Diodes. Adv. Mater. 25, 6801-6827. 10.1002/adma.201301603.

44. Hasan, M.; Shukla, A.; Ahmad, V.; Sobus, J.; Bencheikh, F.; McGregor, S. K. M.; Mamada, M.; Adachi, C.; Lo, S. C.; Namdas, E. B. (2020). Exciton-Exciton Annihilation in Thermally Activated Delayed Fluorescence Emitter. Adv. Funct. Mater. 30 , 2000580. 10.1002/adfm.202000580.

45. Chai, J.-D.; Head-Gordon, M. (2008). Long-range corrected hybrid density functionals with damped atom-atom dispersion corrections. Phys. Chem. Chem. Phys. 10, 6615-6620. 10.1039/B81018gB.

46. Frisch, M. J.; Trucks, G. W.; Schlegel, H. B.; Scuseria, G. E.; Robb, M. A.; Cheeseman, J. R.; Scalmani, G.; Barone, V.; Petersson, G. A.; Nakatsuji, H., et al. Gaussian 16 Rev. C.01, Wallingford, CT, 2016. 